\title{
Insulin sensitivity and cardiovascular risk factors in hypertensive and normotensive native Ghanaians
}

\author{
A. G. B. Amoah ${ }^{1}$, D. P. Schuster ${ }^{2}$, T. Gaillard ${ }^{2}$, K. Osei ${ }^{2}$ \\ ${ }^{1}$ Diabetes Research Laboratory, Department of Medicine and Therapeutics, University of Ghana Medical School, Accra, Ghana \\ ${ }^{2}$ Division of Endocrinology and Metabolism, Department of Internal Medicine, The Ohio State University Hospitals, Columbus, \\ Ohio, USA
}

\begin{abstract}
Aims/Hypothesis. The objective of this study was to examine the relationships among insulin and insulin sensitivity and risk factors for cardiovascular diseases in native Ghanaians with and without hypertension.

Methods. We measured the anthropometric parameters, systolic and diastolic blood pressure, fasting serum triglycerides, cholesterol and high-density lipoprotein cholesterol and fasting and stimulated glucose, insulin and C-peptide of 200 Ghanaian subjects, who were between 25 to 74 years of age, and residing in the Accra Metropolitan area. Serum glucose, C-peptide and insulin concentrations were measured at baseline (fasting) and also $2 \mathrm{~h}$ after $75 \mathrm{gm}$ oral glucose drink. Homeostasis model assessment was used to measure insulin resistance. Hypertension was defined as a blood pressure higher than 140/90 $\mathrm{mmHg}$.

Results. There were 53 subjects with hypertension (HBP) and 147 subjects with normal blood pressure (NBP). The mean BMI, waist circumference and waist-to-hip circumference ratio for $\mathrm{HBP}$ and NBP subjects were $27.4 \pm 0.8,24.8 \pm 0.4 \mathrm{~kg} / \mathrm{m}^{2} ; 89.8 \pm 11.7$, $81.1 \pm 0.9 \mathrm{~cm}$; and $0.87 \pm 0.08,0.82 \pm 0.08$ respectively, $(p<0.05)$. The fasting and 2-h plasma glucose concen-
\end{abstract}

Received: 5 August 2002 / Revised: 4 March 2003

Published online: 18 June 2003

(C) Springer-Verlag 2003

Corresponding author: A. G. B. Amoah, Diabetes Research Laboratory, Department of Medicine and Therapeutics, University of Ghana Medical School, Post Office Box 4236, Accra, Ghana

E-mail: agbamoah@ghana.com

Abbreviations: HBP, hypertension; NBP, normal blood pressure; HOMA \% BC, HOMA derived Beta-cell function; CVD, cardiovascular disease; HOMA, homeostasis model assessment; HOMA-IR, homeostasis model assessment derived insulin resistance. trations in HBP and NBP subjects were 5.5 \pm 0.2 , $7.2 \pm 0.3 \mathrm{mmol} / \mathrm{l}$ and $5.2 \pm 01,6.8 \pm 0.2 \mathrm{mmol} / 1 \mathrm{respec}-$ tively $(p>0.05)$. The corresponding fasting and $2-\mathrm{h}$ insulin concentrations were $10.0 \pm 0.7,8.0 \pm 0.4 \mathrm{uU} / \mathrm{ml}$ and $47.3 \pm 3.7,37.3 \pm 2.5 \mathrm{uU} / \mathrm{ml}$ respectively $(p<0.05)$. The insulin resistance index (HOMA-IR) in the HBP and the NBP groups were $2.49 \pm 0.2$ and $1.95 \pm 0.13$ $(p<0.05)$. The two groups had similar fasting and stimulated C-peptide, lipids and HDL concentrations. Correlations were found between blood pressure and the concentrations of lipids, HDL, fasting and stimulated insulin and C-peptide, and between fasting insulin and HOMA-IR with lipids and HDL concentrations. On multiple regression analysis, fasting insulin and HOMA-IR did not influence blood pressure variations significantly.

Conclusions/interpretation. We found clustering of hyperinsulinaemia, insulin resistance and truncal obesity in hypertensive Ghanaian subjects but dissociation between insulin resistance, hypertension and atherogenic lipid and lipoprotein profile. [Diabetologia (2003) 46:949-955]

Keywords Insulin sensitivity, blood pressure, lipids, lipoproteins, hypertension, ghanaians, insulin resistance.

The high prevalence and increasing burden of cardiovascular diseases (CVD) in developing countries has recently been highlighted in the Global Burden of Disease study [1, 2]. Insulin resistance and hyperinsulinaemia are recognized risk factors for cardiovascular disease (CVD) [3, 4, 5]. The resistance to the action of insulin is also accompanied by compensatory increase in insulin secretion by the beta cells and hyperinsulinaemia to maintain normoglycaemia. The insulin resistance and other CVD risk factors seem to be increasing worldwide [6]. Whether this also accounts for the increasing prevalence of hypertension, and 
cerebrovascular accidents in sub-Saharan Africa is uncertain $[7,8,9,10]$.

Several disorders are associated with insulin resistance and hyperinsulinaemia and tend to cluster in the same individual $[5,11,12,13,14]$. They include high serum triglycerides, low HDL, high blood pressure, increased plasminogen activator inhibitor (PAI-1), hyperfibrinogenemia, increased C-reactive protein (CRP), glucose intolerance and central or upper body obesity $[5,11,12,13,14]$. These disorders, which are also risk factors for cardiovascular disease, together are referred to as syndrome $\mathrm{X}$, metabolic syndrome or insulin resistance syndrome $[5,11,12,13,14]$. It has been suggested that insulin stimulates renal sodium absorption, cation transport, sympathetic system stimulation, lipoprotein transport to the endothelium, intimal and medial areas of the vessel, and proliferation of vascular smooth muscle $[15,16]$.

Recently, it has been questioned whether the metabolic syndrome as originally described [5] prevails in most or all populations $[16,17,18,19]$. The prevalent rates of different aspects of the syndrome vary in different racial and ethnic populations. For example, African Americans, especially women, are in general more obese and hypertensive, and have greater insulin resistance with hyperinsulinaemia but lower serum fasting triglycerides and higher high-density lipoprotein cholesterol concentrations than white Americans $[16,17,18,19,20,21]$. Also no relationship between insulin resistance and blood pressure has been found in African-American subjects [17, 18]. Whether these findings extend to native West Africans who share a common genetic heritage with African-Americans and Afro-Caribbean people remain uncertain.

Ghana is a tropical country in West Africa with a surface area of $238.533 \mathrm{~km}^{2}$, an estimated population of 19.7 million, a gross domestic product (GDP) of 7.4 billion US dollars and per capita GDP of 412 US dollars. The Accra Metropolitan area has the largest urban population (1.6 million) [22]. In a recent study, we showed that non-diabetic Ghanaian subjects residing in USA or their native country manifest insulin resistance and hyperinsulinaemia, similar to African American subjects [23, 24]. Similarly, serum insulin concentrations in the Ghanaian subjects are reportedly higher than those of white Americans [17]. These observations are of great interest as African-Americans share ethnogenetic links with West Africans. However, the relationship between serum insulin, insulin resistance and components of the metabolic syndrome and hypertension status in Ghanaian subjects living in Ghana has not been examined. Therefore, we sought to examine the relationships of blood pressure and lipids and lipoproteins and obesity indices in a community sample of 200 free-living, native Ghanaians from the Accra Metropolitan area of Ghana.

\section{Subjects and methods}

Subjects. The study population comprised of 200 adults (age range 25-74 years), selected from a random community sample (cluster) from the Accra Metropolitan area of Ghana. Subjects with fasting plasma glucose of less than $3.5 \mathrm{mmol} / \mathrm{l}$ or who were on exogenous insulin or anti-diabetic medication were excluded from the study. The subjects were non-smokers with no previous diagnosis of hypertension or diabetes, and they were not on medications that interfere with insulin action, or glucose and insulin responses. They were allowed their usual diet which is relatively high in carbohydrates (i.e. $>250 \mathrm{~g}$ carbohydrate per day) for the 3 days prior to testing.

Study protocol. After an overnight fast of 10 to $12 \mathrm{~h}$, anthropometric measurements were carried out on subjects in light clothing and without shoes. Body weight was measured with a heavy duty Seca 770 floor digital scale (Seca, Hamburg, Germany) to the nearest $0.1 \mathrm{~kg}$. Height was measured with a height stadiometer to the nearest $0.1 \mathrm{~cm}$. Body mass index was calculated from the weight and height measurements. Waist and hip girth were measured in duplicate with a non-elastic plastic measuring tape to the nearest $0.1 \mathrm{~cm}$. The waist measurement was taken at the mid-point between the lower rib margin and the iliac crest at the end of gentle expiration. The hip girth was obtained at the level of the greater trochanter. The mean of the duplicate data was used to determine the waist girth and WHR. After at least 10 -min rest, two blood pressure readings were taken by trained nurses using a zero-centered sphygmomanometer.

Metabolic protocol. Fasting blood was drawn for plasma glucose, serum cholesterol, triglycerides, high-density lipoprotein, insulin and C-peptide concentrations. Each subject then ingested $75 \mathrm{gm}$ of anhydrous glucose dissolved in $250 \mathrm{mls}$ of water over 2 min. Blood samples were obtained $2 \mathrm{~h}$ after this for determination of plasma glucose, serum insulin and C-peptide concentrations.

Analytical methods. Serum triglyceride, cholesterol and HDL concentrations were measured by enzymatic methods and glucose by glucose oxidase (Randox Laboratories Limited, Crumlin, UK) on a chemistry autoanalyzer (Erba Smartlab, Mumbai, India). The inter-assay coefficient of variation for normal and increased plasma glucose controls were $1.4 \%$ and $2.4 \%$ respectively, the corresponding intra-assay coefficient of variation being $1.8 \%$ and $1.4 \%$ respectively. The serum insulin and C-peptide concentrations of each individual were determined by a standard double antibody radioimmunoassay technique with the sensitivity of the insulin assay being less than $2.5 \mathrm{uU} / \mathrm{ml}$. Intra- and inter-assay coefficients of variation were $5 \%$ and $10 \%$, respectively. The lower limit of the C-peptide assay was $0.20 \mathrm{nmol} / \mathrm{ml}$ and the intra-assay and inter-assay coefficient of variation, were $7 \%$ and $13 \%$, respectively.

Calculation and statistical analyses. Analysed results are expressed as means \pm SEM, unless otherwise stated. High blood pressure was defined as $\geq 140 / 90 \mathrm{mmHg}$ in accordance with the 1997 Joint National Committee Criteria [25]. Insulin resistance index was calculated using the homeostasis model assessment (HOMA) [26]. HOMA insulin resistance (HOMA-IR) was calculated in accordance with the folowing formula: fasting insulin $(\mathrm{uU} / \mathrm{ml}) \times$ fasting plasma glucose $(\mathrm{mmol} / \mathrm{l}) / 22.5$. Beta-cell function (HOMA \%B) was determined from the formula: $20 \times$ fasting insulin $(\mathrm{uU} / \mathrm{ml}) /$ fasting glucose-3.5. HOMA is more suitable and convenient for such epidemiologic studies than the gold standard but more laborious euglycemic clamp [27] or frequently sampled intravenous glucose tolerance test [28, 29]. 
Table 1. Clinical and metabolic characteristics of Ghanaian subjects by sex

\begin{tabular}{|c|c|c|c|c|}
\hline & Males $(n=87)$ & Females $(n=113)$ & $p$ value & All Subjects \\
\hline Age (years) & $40.8 \pm 1.3$ & $41.5 \pm 1.3$ & $>0.05$ & $41.2 \pm 1.3$ \\
\hline BMI $\left(\mathrm{kg} / \mathrm{m}^{2}\right)$ & $24.2 \pm 0.4$ & $26.6 \pm 0.5$ & $<0.001$ & $25.5 \pm 0.3$ \\
\hline WHR & $0.88 \pm 0.01$ & $0.80 \pm 0.01$ & $<0.01$ & $0.84 \pm 0.01$ \\
\hline Systolic BP (mmHg) & $131 \pm 2$ & $127 \pm 2$ & $>0.05$ & $128 \pm 2$ \\
\hline Diastolic. BP (mmHg) & $78 \pm 1$ & $75 \pm 1$ & $>0.05$ & $76 \pm 1$ \\
\hline Cholesterol $(\mathrm{mmol} / \mathrm{l})$ & $4.68 \pm 0.06$ & $4.94 \pm 0.11$ & $>0.05$ & $4.83 \pm 0.08$ \\
\hline Triglycerides (mmol/l) & $0.50 \pm 0.03$ & $0.45 \pm 0.02$ & $>0.05$ & $0.47 \pm 0.02$ \\
\hline $\operatorname{HDL}(\mathrm{mmol} / \mathrm{l})$ & $1.06 \pm 0.06$ & $1.10 \pm 0.05$ & $>0.05$ & $1.08 \pm 0.04$ \\
\hline Fasting C-peptide(ng/ml) & $1.08 \pm 0.11$ & $1.22 \pm 0.07$ & $>0.05$ & $1.16 \pm 0.06$ \\
\hline Fasting serum insulin (uU/ml) & $8.4 \pm 0.5$ & $8.64 \pm 0.4$ & $>0.05$ & $8.5 \pm 0.9$ \\
\hline HOMA-IR & $2.10 \pm 0.16$ & $2.09 \pm 0.15$ & $>0.05$ & $2.10 \pm 0.11$ \\
\hline
\end{tabular}

Values are means $\pm \mathrm{SEM}$

Unless indicated otherwise, the statistical package SPSS 10.0 for Windows (SPSS, Chicago, Ill., USA) was used for analyses. Multiple regression and correlation coefficients were calculated using least squares method. The Student's unpaired two-sided $t$ test was used to compare means between two groups and the one-way ANOVA was used to compare more than two groups of variables. Statistical significance was considered as probability value less than 0.05 .

The study was approved by the Ethics Review Committee of the University of Ghana Medical School and complied with the Helsinki Declaration of 1975 (revised in 1983) on human experimentation. Informed consent was obtained from each subject before participation.

\section{Results}

The clinical characteristics of the subjects are shown in Table 1. The study comprised of 200 adults (males:females $=87: 113$ ), mean age $41.2 \pm 1.3$ years. Except for the body mass index and waist-to-hip circumference ratio which were lower in the women than men, no significant differences were observed in the other parameters (Table 1).

Table 2 shows some clinical characteristics of hypertensive (HBP) and normotensive (NBP) subjects. There were 147 (female:male=1.3:1.0) subjects with normal blood pressure and 53 (female:male=1.3:1.0) with hypertension. The prevalence of hypertension in this Ghanaian cohort was $26.5 \%$. The HBP subjects compared to NBP subjects were older, heavier and had more central obesity $(p<0.05)$.

Table 3 shows the metabolic risk factor profile for the normotensive and hypertensive subjects. There were no significant differences between the two groups for the concentrations of fasting glucose, 2-h glucose, fasting lipids, fasting and 2-h C-peptide and HOMA \%B scores. In contrast, fasting insulin, 2-h serum insulin and HOMA-IR concentrations were higher in the HBP than in the NBP group.
Table 2. Clinical characteristics of subjects by blood pressure status

\begin{tabular}{lll}
\hline Parameters & NBP & HBP \\
\hline Sex ratio (men/women) & $64 / 83$ & $23 / 30$ \\
Age (years) & $37.4 \pm 0.9$ & $51.5 \pm 1.8^{*}$ \\
BMI $\left(\mathrm{kg} / \mathrm{m}^{2}\right)$ & $24.8 \pm 0.4$ & $27.4 \pm 0.8^{*}$ \\
Waist $(\mathrm{cm})$ & $81.1 \pm 0.9$ & $89.8 \pm 11.7^{*}$ \\
WHR & $0.82 \pm 0.08$ & $0.87 \pm 0.08^{*}$ \\
Systolic BP $(\mathrm{mmHg})$ & $118 \pm 1$ & $157 \pm 3^{*}$ \\
Diastolic BP $(\mathrm{mmHg})$ & $71 \pm 1$ & $91 \pm 2^{*}$
\end{tabular}

Values are means \pm SEM; NBP, normal blood pressure; HBP, high blood pressure; $* p>0.05$ HTN vs. NTN

Table 3. Metabolic risk factor profile of subjects by blood pressure status

\begin{tabular}{lcc}
\hline & NBP & HBP \\
\hline Fasting plasma glucose (mmol/l) & $5.2 \pm 0.1$ & $5.5 \pm 0.2^{\mathrm{b}}$ \\
2-h Plasma glucose (mmol/l) & $6.8 \pm 0.2$ & $7.2 \pm 0.3^{\mathrm{b}}$ \\
Cholesterol (mmol/l) & $4.7 \pm 0.1$ & $5.2 \pm 0.2^{\mathrm{b}}$ \\
HDL (mmol/l) & $1.08 \pm 0.04$ & $1.08 \pm 0.06^{\mathrm{b}}$ \\
Triglicerydes (mmol/l) & $0.45 \pm 0.02$ & $0.52 \pm 0.03^{\mathrm{b}}$ \\
Fasting serum insulin (uU/ml) & $8.0 \pm 0.4$ & $10.0 \pm 0.7^{\mathrm{a}}$ \\
2-h Iinsulin (uU/ml) & $37.3 \pm 2.5$ & $47.3 \pm 3.7^{\mathrm{a}}$ \\
Fasting C-peptide (ng/ml) & $1.10 \pm 0.07$ & $1.32 \pm 0.11^{\mathrm{b}}$ \\
2-h C-peptide (uU/ml) & $4.50 \pm 0.24$ & $5.26 \pm 0.36^{\mathrm{b}}$ \\
HOMA-IR & $1.95 \pm 0.13$ & $2.49 \pm 0.20^{\mathrm{a}}$ \\
HOMA-\%BC & $26.8 \pm 1.2$ & $33.7 \pm 2.7^{\mathrm{a}}$ \\
\hline
\end{tabular}

Values are means \pm SEM; ${ }^{\mathrm{a}} p<0.05$; ${ }^{\mathrm{b}} \mathrm{NBP}$ vs HBP

Table 4 shows the zero order and fourth order partial correlation of systolic and diastolic blood pressures with cardiovascular risk factors. Age and anthropometric indices had the highest correlation with systolic and diastolic blood pressure. The relationship between blood pressure and the metabolic parameters, 
Table 4. A Partial correlation of systolic and diastolic blood pressures with cardiovascular risk factors. B Partial correlation of fasting serum insulin and HOMA-IR and cardiovascular risk factors

\begin{tabular}{|c|c|c|c|c|}
\hline \multirow{2}{*}{$\mathbf{A}$} & \multicolumn{4}{|c|}{ Correlation coefficients $(r)$} \\
\hline & \multicolumn{2}{|l|}{ Zero order Partials } & \multicolumn{2}{|c|}{ Fourth order partials } \\
\hline Age & 0.506 & 0.375 & & \\
\hline BMI & 0.314 & 0.368 & & \\
\hline Waist girth & 0.377 & 0.458 & & \\
\hline 2-h plasma glucose $\mathrm{mmol} / \mathrm{l}$ & 0.148 & 0.130 & -0.038 & -0.018 \\
\hline Triglycerides & 0.234 & 0.284 & 0.058 & 0.086 \\
\hline Cholesterol & 0.203 & 0.135 & 0.072 & -0.002 \\
\hline HDL & -0.022 & 0.008 & -0.052 & -0.037 \\
\hline Fasting insulin & 0.226 & 0.206 & 0.068 & -0.007 \\
\hline B & Fasting Insulin & HOMA-IR & Fasting Insulin & HOMA-IR \\
\hline Age & 0.214 & 0.231 & & \\
\hline BMI & 0.471 & 0.360 & & \\
\hline Waist girth & 0.478 & 0.379 & & \\
\hline WHR & 0.247 & 0.211 & & \\
\hline Triglycerides & 0.373 & 0.396 & 0.224 & 0.282 \\
\hline Cholesterol & 0.270 & 0.250 & 0.155 & 0.153 \\
\hline HDL & 0.042 & 0.026 & 0.004 & -0.002 \\
\hline
\end{tabular}

Table 5. A Multiple regression analysis of systolic and diastolic blood pressure and cardiovascular risk factors. Model Summary (Outcome: Systolic BP)*. B Multiple regression analysis of systolic and diastolic blood pressure and cardiovascular risk factors. Model Summary (Outcome: diastolic BP)*

\begin{tabular}{lllll}
\hline Model & $r$ & $r^{2}$ & Adjusted $r^{2}$ & $\begin{array}{l}\text { Standard error } \\
\text { of estimate }\end{array}$ \\
\hline $\mathbf{A}$ & & & & \\
$1^{\mathrm{a}}$ & .532 & .283 & .268 & 19.34 \\
$2^{\mathrm{b}}$ & .577 & .332 & .283 & 19.14 \\
$\mathbf{B}$ & & & & \\
$1^{\mathrm{a}}$ & .498 & .248 & .232 & 11.39 \\
$2^{\mathrm{b}}$ & .521 & .271 & .217 & 11.50 \\
\hline
\end{tabular}

a Predictors: (Constant), WHR, BMI, age, waist

b Predictors: (Constant), WHR, BMI, age, waist, fasting plasma glucose, 2-h plasma glucose, HDL, fasting cholesterol, fasting triglycerides, fasting C-peptide, plasma glucose insulin, fasting insulin, plasma glucose, C-peptide, HOMA-IR, HOMA \% BC

$*$ ANOVA $p<0.001$

including the insulin sensitivity index, HOMA-IR, was weak (zero order partial correlation); the association became weaker still, when adjustments were made for age, BMI, waist girth and WHR (fourth order partial correlation).
We found weak correlations between fasting insulin, HOMA-IR and the anthropometric parameters and serum triglycerides, cholesterol and HDL (zero order partials). When adjustments were made for Age, BMI, waist girth and WHR (fourth order partials), the association of fasting insulin and HOMA-IR with triglycerides, cholesterol and HDL became weaker still (Table 5). To examine the influence of the risk factors as predictors of blood pressure, we developed models from multiple regression analysis (Table 5). HOMAIR accounted for only 2.83 and $2.48 \%$ variance in systolic and diastolic blood pressure, respectively (Table 5).

\section{Discussion}

Our study examined the relationship between insulin, insulin resistance on the one hand and blood pressure and atherogenic lipids and lipoproteins on the other in native Ghanaian people. We found $26.5 \%$ of the randomly selected Ghanaian cohort to be hypertensive. Although this figure is comparable to those reported $[8,9,10,11]$ in Sub-Saharan African Black people, we believe this might not represent the true prevalence of hypertension in Ghanaian people since we studied only a small sample of individuals residing in the Accra Metropolitan area. Of note, this rate of hyper- 
tension in the native Ghanaian people is similar to those of African-Americans [31]. Plasma glucose concentrations during fasting and $2 \mathrm{~h}$ after oral glucose challenge were similar in the hypertensive (HBP) and normotensive (NBP) subjects. In contrast, the mean serum insulin concentrations and HOMA-IR were greater in the hypertensive subjects when compared to the normotensive Ghanaians. There was, however, no association between blood pressure and some of the cardiovascular risk factors.

A major component of the Metabolic Syndrome is altered lipid metabolism. Specifically, patients with the metabolic syndrome manifest high serum triglycerides and low HDL concentrations while serum cholesterol tends to be normal $[4,5]$. In our study, the serum triglycerides, HDL and cholesterol concentrations were all normal in subjects with hypertension. We have reported previously that non-hypertensive Ghanaian subjects with impaired fasting glucose, impaired glucose tolerance and Type 2 diabetes [30] had normal lipid and lipoprotein concentrations which were similar to those of African-Americans and AfroCaribbeans [29, 31, 32].

Previous studies have shown that increased waist circumference, WHR and BMI are associated with increased insulin resistance, hyperinsulinaemia, low HDL and high serum triglyceride and glucose intolerance in the Caucasian [11, 12, 14, 15, 16] and MexicanAmericans [13]. In our study, subjects with hypertension had greater central obesity and higher fasting serum insulin and 2-h serum insulin concentrations than subjects with normal blood pressure despite identical plasma glucose concentrations. These findings are consistent with those in other non-African populations $[4$, $5,12,13,14,15,16,17,18]$. In our study, however, the greater serum insulin responses and insulin resistance in subjects with high blood pressure was associated with normal serum cholesterol, triglycerides and HDL concentrations. The favorable anti-atherogenic profiles in our hypertensive subjects, was indeed comparable to those in our normotensive subjects. Thus, the insulinresistant hypertensive Ghanaian subjects extend the favorable lipid profiles found in normotensive Ghanaian subjects [30] African-Americans [20, 21, 29] and AfroCarribeans $[31,32]$ when compared to other populations such as Caucasians.

The mechanism of the insulin resistance with the concomitant greater serum insulin concentrations in the Ghanaian and the Sub-Saharan African populations in general with high blood pressure is not known. There is a strong evidence to suggest insulin resistance is genetically determined with a strong familial and environmental component $[33,34,35,36$, 37]. Insulin resistance has a predilection for certain ethnic groups [13, 16, 17, 24, 36, 37]. In this regard, previous studies found greater hyperinsulinaemia and insulin resistance in African-Americans [18, 24], Ghanaians [37], Afro-Caribbeans [32, 33], Pima Indians
[16], and Mexican-Americans [13, 37] compared to Caucasians. In a previous study in Ghanaian subjects living in their native country, we showed an increased insulin resistance in the first degree relatives of patients with Type 2 diabetes, compared to those with no family history of Type 2 diabetes, suggesting both genetic or familial contributions [37]. In the present study our subjects with high blood pressure were older, and had more truncal obesity as evidenced by the greater waist girth and WHR, compared to normotensive subjects. Thus, it is possible that the ageing and increased waist girth and WHR, the surrogates for intra-abdominal visceral adiposity, could partly account for the differences in the insulin resistance in the hypertensive and normotensive Ghanaian subjects. Insulin resistance, however, accounted for only 2.8 and $2.5 \%$ of the variation in systolic and diastolic blood pressure respectively, in our subjects. Also we found only a weak relationship between insulin resistance and waist girth and WHR. The statistical significance of these findings is therefore uncertain. Our data suggests that other factors not assessed in our study e.g. genetics, physical activity etc. probably accounted for the differences in the insulin resistance in the normotensive and hypertensive Ghanaian cohort.

Finally, apart from genetic inheritence, there are several environmental factors that can influence the development of hypertension. In particular, low birth weight with rapid early childhood growth and weight gain, has been implicated in the accelerated development of several diseases $[38,39]$. In this regard several studies [40, 41, 42] have reported that small babies, for their for gestational age, have accelerated catch-up growth, high blood pressure and insulin resistance in black South African children and/or adults. Previous studies have also established that low birth weight babies who acquire rapid weight gain as infants, manifest insulin and insulin resistance syndrome (and perhaps in later adult life) compared with those with low birth weight who had a slow progression in weight gain in black African children living in South Africa [40]. These previous findings in Sub-Saharan African people would support the "fetal origin of diseases in the old age" proposed by another study [38].

Implications of present study for Africa. The increasing rates of insulin resistance, hypertension, cardiovascular disease risk factors and diabetes in SubSaharan Africans in epidemiological health transitioning have serious economic implications for these countries $[8,9,10]$. Most countries in Africa with generally low income, can least afford the human toll and economic burden from large numbers of patients with non-communicable diseases such as Type 2 diabetes, cerebrovascular accidents, and congestive heart failure as well as end stage renal diseases on account of limited resources [22]. Thus, accurate but simple and less expensive techniques to examine the association of insulin concentrations and insulin resistance 
and cardiovascular disease risks could contribute to the understanding of the mechanism of non-communicable cardiovascular diseases and diabetes in SubSaharan African countries.

In conclusion, we examined the relationship of insulin, insulin resistance and conventional anthropometric and metabolic CVD risk factors in Ghanaian subjects residing in Ghana. While we found truncal obesity, higher serum insulin concentrations and greater insulin resistance in subjects with high blood pressure, there was only a weak relationship between serum insulin and insulin resistance and blood pressure and lipids and lipoproteins similar to those found in South-African black, African-Americans and AfroCaribbean people. Thus, the role of insulin resistance and hyperinsulinaemia in the development of cardiovascular diseases deserves further investigation in long-term prospective studies in Ghanaian and other populations in the Sub-Saharan region.

Acknowledgements. We wish to thank Rev. Prof. S. Ayettey for his helpful comments, and to thank Charles Oppong and Henry Edo, Diabetes Research Laboratory, University of Ghana Medical School, Accra, Ghana, and staff of the Core Laboratory at The Ohio State University College of Medicine and Public Health, Columbus, Ohio for their technical support. Financial assistance from the National Diabetes Management and Research Centre and from GCRC-RR034 is acknowledged.

The source of funding of this study was provided by the National Diabetes Management and Research Centre, Accra, Ghana and the Division of Endocrinology and Metabolism, The Ohio State University Hospitals, Columbus, Ohio, USA.

\section{References}

1. Murray CJL, Lopez AD (1996) Global health statistics: global burden of disease and injury series. Harvard School of Public Health, Boston, pp 23-26

2. The World Health Organization, Study Group on Diabetes Mellitus (1999) Making a difference. World Health Organization, Geneva

3. Wing RR, Bunker CH, Kuller LH, Mathews KA (1989) Insulin, body mass index, and cardiovascular risk factors in premenopausal women. Arteriosclerosis 9:479-484

4. Manolio TA, Savage PJ, Burge GL et al. (1990) Association of fasting insulin with blood pressure and lipids in young adults. The CARDIA Study. Arteriosclerosis 10:430-436

5. Reaven GM (1988) Role of insulin resistance in human disease. Diabetes 37:1595-1607

6. Seidell JC (2000) Obesity, insulin resistance and diabetes-a world wide epidemic. Br J Nutr 83:S5-S8

7. Burdon J (1998) Hypertension treatment and control in Sub-Saharan Africa. Figure of $\$ 1800$ per life saved seems optimistic. BMJ 317:76-77

8. Walker R, Unwin N, Alberti KGMM (1998) Hypertension treatment and control in Sub-Saharan Africa. Burden of cerebrovascular disease will increase as more people survive to old age. BMJ 317:76-77

9. Cooper RS, Rotimi CN, Ataman S et al. (1998) The prevalence of hypertension in seven populations of West African origin. Am J Public Health 87:160-168

10. Walker R (1994) Hypertension and stroke in sub-Saharan Africa. Trans R Soc Trop Med Hyg 88:609-661
11. Cooper RS, Rotimi CN, Ataman SL et al. (1997) Prevalence of hypertension in seven populations of African origin. Am J Public Health 87:160-960

12. Ferrannini E, Haffner SM, Mitchell BD, Stern MP (1991) Hyperinsulinemia: the key feature of a cardiovascular and metabolic syndrome. Diabetologia 34:416-422

13. Haffner SM, Vldez RA, Hazuda HP, Mitchell BD, Morales PA, Stern MP (1992) Prospective analysis of he insulinresistance syndrome (syndrome X). Diabetes 41:715722

14. Schwartz SM (1995) The intimal: soil for atherosclerosis and restenosis. Circ Res 77:445-465

15. DeFronzo RA, Ferrannini E (1991) Insulin resistance: a multifaceted syndrome responsible for NIDDM, obesity, hypertension, dyslipidaemia and atherosclerotic cardiovascular disease. Diabetes Care 14:173-194

16. Saad MF, Lillioja S, Nyomba BL et al. (1991) Racial differences in the relation between blood pressure and insulin resistance. N Engl J Med 324:733-739

17. Osei K, Schuster DP (1996) Effects of race and ethnicity and Ethnicity on insulin sensitivity, blood pressure, and heart rate in 3 ethnic populations; comparative studies in African-Americans, African immigrants (Ghanaians), and white Americans using ambulatory blood pressure monitoring. Am J Hypertension 9:1157-1164

18. Okosun IS, Cooper RS, Prewitt TE, Charles CN (1998) The relationship of central adiposity to components of the insulin resistance syndrome in a biracial US Population Sample. Ethnicity Dis 9:218-228

19. Toft I, Bonaa KH, Jenssen T (1998) Insulin resistance in hypertension is associated with body fat rather than blood pressure. Hypertension 32:115-122

20. Metcalf PA, Sharrett AR, Folsom AR et al. (1998) African American-white differences in lipids, lipoproteins and apolipoprotein, by educational attainment, among middle-aged adults: the Atherosclerosis Risk in Communities Study. Am J Epidemiol 148:750-760

21. Cowie CC, Howard BV, Harris MI (1991) Serum lipoproteins in African Americans and whites with non-insulindependent diabetes in the US population. Circulation 90:1185-1193

22. World Statistics Pocketbook (2000) Department of Economic and Social Affairs, United Nations, New York

23. Osei K, Schuster DP (1995) Metabolic characteristics of African descendants: a comparative study of AfricanAmericans and Ghanaian immigrants using minimal model analysis. Diabetologia 38:1103-1109

24. Osei K, Schuster DP (1994) Ethnic differences in secretion, sensitivity and hepatic extraction of insulin in black and white Americans. Diabet Med 11:755-762

25. Joint National Committee on Prevention, Detection, Evaluation, and Treatment of High Blood Pressure (1998) The Sixth Report of the Joint National Committee on Prevention, Detection, Evaluation, and Treatment of High Blood Pressure. Arch Intern Med 157:2413-2442

26. Mathews DR, Hosker JP, Rudenski AS, Taylor BA, Treacher DF, Turner RC (1985) Homeostasis model assessment insulin resistance and beta cell function from fasting plasma glucose and insulin concentrations in man. Diabetologia 28:412-419

27. DeFronzo RA, Tobin JD, Andres R (1979) The glucose clamp technique: a method for the quantification of beta cell sensitivity to glucose and of tissue sensitivity to insulin. Am J Physiol 237:E214-E223

28. Bergman RN, Phillips LS, Cobelli C (1981) Physiological evaluation of factors controlling glucose tolerance in man. J Clin Invest 68:1456-1467 
29. Osei K. Gaillard T, Schuster DP (1999) Cardiovascular risk factors in African Americans with varying degrees of glucose intolerance. Diabetes Care 22:1588-1590

30. Amoah AGB (2002) Undiagnosed diabetes and impaired glucose regulation in adult Ghanaians using the ADA and WHO diagnostic criteria. Diabetologia 39:7-13

31. Fontbonne A, Papoz L, Eschwege E, Roger M, Saint-Paul M, Simon D (1992) Features of insulin resistance syndrome in men from French Caribbean Islands. The Telecom Study. Diabetes 41:1385-1389

32. Cruickshank JK, Mbanya JC, Wilks R, Balku B, McFarlene-Anderson N, Forester T (1998) Sick genes, sick individuals and sick populations with chronic disease? The emergence of diabetes and high blood pressure in African-origin populations. Int J Epidemiol 30:111-117

33. Young CA, Kumar S, Young MJ, Boulton AJ (1995) Excess maternal history of diabetes in Caucasian and Afroorigin non-insulin-dependent diabetic patients: maternal factors in disease transmission. Diab Res Clin Pract 28:47-49

34. Osei K, Cottrell DA (1994) Minimal model analyses of insulin sensitivity and glucose dependent glucose disposal in black and white Americans: a study of persons at risk for type 2 diabetes. Eur J Clin 24:843-850

35. Rotimi CN, Dunston GM, Berg K et al. (2001) In search of susceptibility genes for type diabetes in West Africa: design and results of the first of the AADM Study. Ann Epidemiol 11:51-58
36. Haffner S, Howard R, Saad P et al. (1997) Insulin sensitivity and acute insulin responses in African Americans, nonHispanic whites and Hispanics and NIDDM: the Insulin Resistance and Atherosclerosis Study 46:63-69

37. Amoah ABG, Owusu SK, Ayittey OM, Schuster DP, Osei K (2001) Minimal model analyses of beta cell secretion, insulin sensitivity and glucose effectiveness in glucose tolerant, first degree relatives of Ghanaian patients. Ethnicity Dis 11:201-210

38. Barker DJP (1992) The fetal origin of disease in old age. Eur J Clin Nutr 46:S3-S9

39. Eriksson JG, Forsen T, Tuomilehto J, Jaddoe VWV, Osmond C, Barker DJP (2002) Effects of size at birth and childhood growth on the insulin resistance syndrome in elderly individuals. Diabetologia 45:342-348

40. Crowther NJ, Cameron N, Trusler J, Gray IP (1997) Association between poor glucose tolerance and rapid postnatal weight gain in seven year old children. Diabetologia 41:1163-1167

41. Levitt NS, Lambert EV, Woods D, Hales N, Andrew R, Seckl JR (2000) Impaired glucose tolerance and elevated blood pressure in low birth weight, nonobese, young South African adults. J Clin Endocrinol Metab 85:46224618

42. Gray IP, Cooper PA, Cory BJ, Toman M, Crowther NJ (2002) The intrauterine environment is a strong determinant of glucose intolerance during the neonatal period even in prematurity. J Clin Endocrinol Metab 87:4252-4256 